\title{
Deucravacitinib, an Oral, Selective Tyrosine Kinase 2 (TYK2) Inhibitor, Versus Placebo and Apremilast in Moderate to Severe Plaque Psoriasis:
}

Efficacy Analysis by Baseline Disease Characteristics From the Phase 3 POETYK PSO-1 and PSO-2 Trials

Joseph F Merola, ${ }^{1}$ Howard Sofen, ${ }^{2}$ Diamant Thaç, ${ }^{3}$ Carle Paul, ${ }^{4}$ Shinichi Imafuku, ${ }^{5}$ Subhashis Banerjee, ${ }^{6}$ Elizabeth Colston, ${ }^{6}$ Jonghyeon Kim, ${ }^{6}$ John Throup, ${ }^{6}$ April W Armstrong ${ }^{7}$

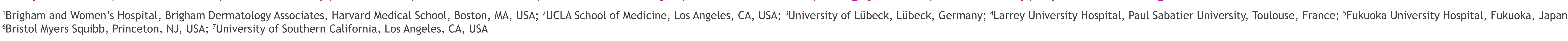

Introduction

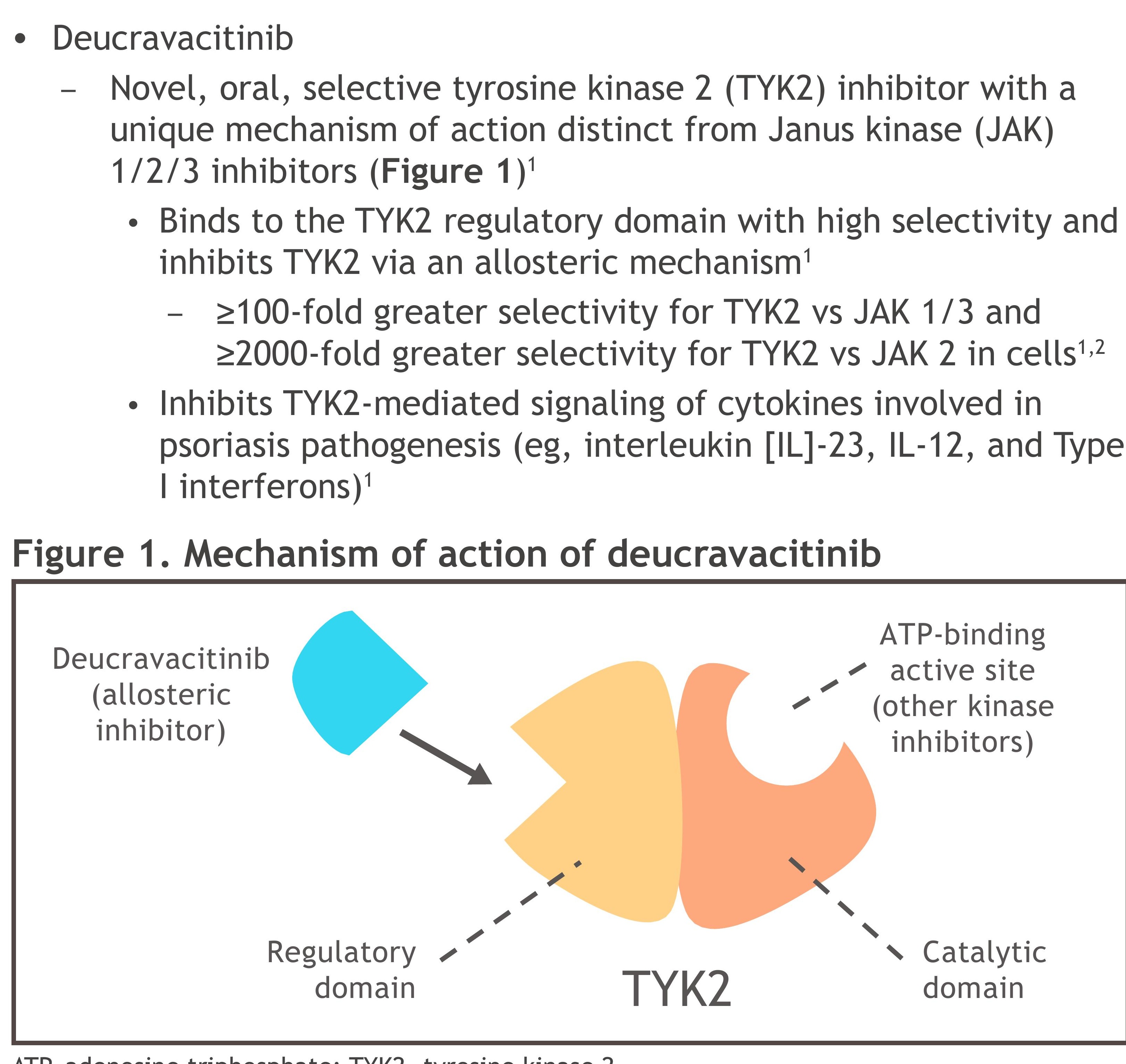

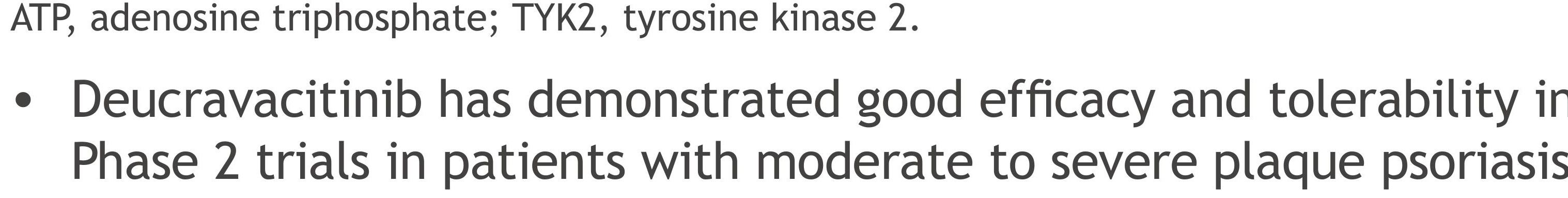

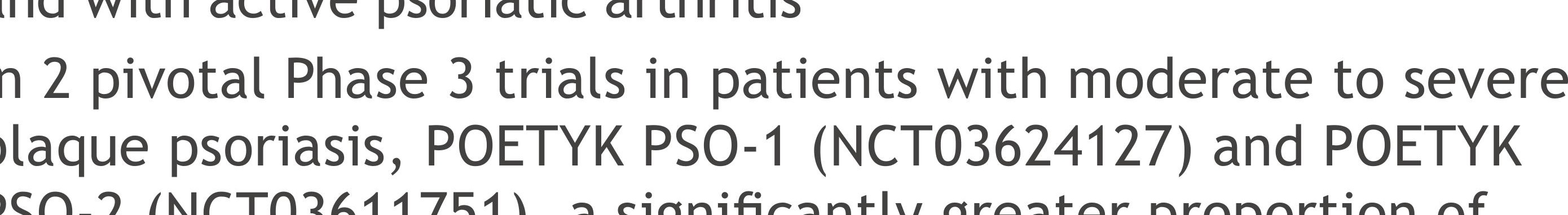

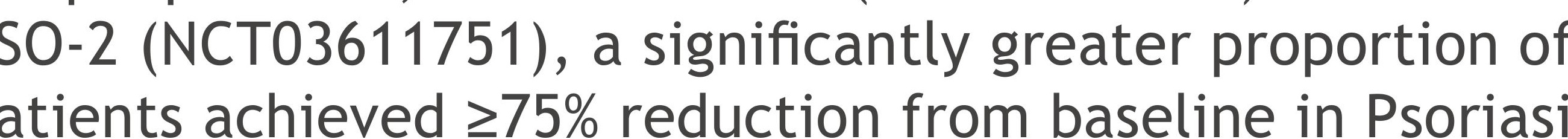

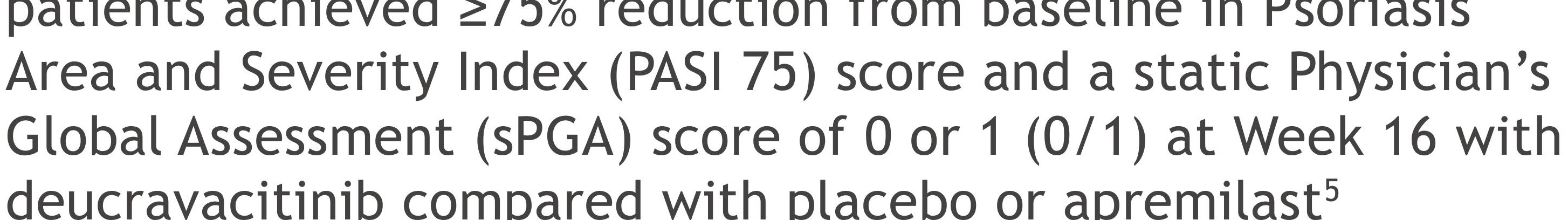

Objective

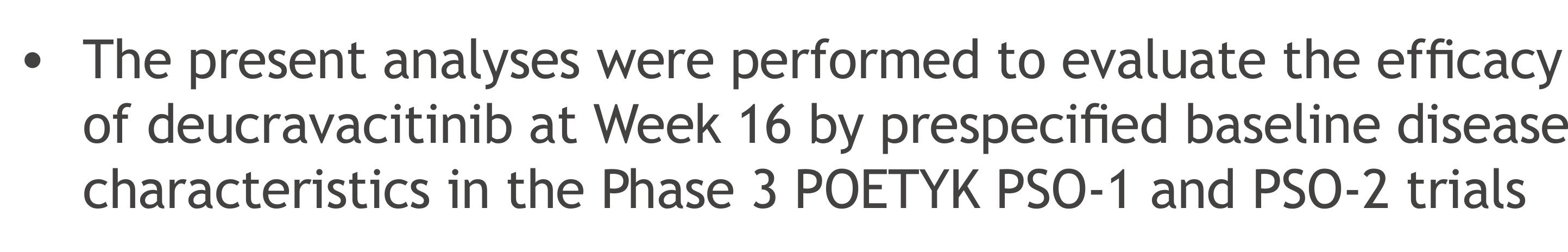

Methods

Key design elements
- The POETYK PSO-1 and PSO-2 study designs are shown in Figure 2

- Adults with chteria

Adults with moderate to severe plaque psoriasis
PASI $\geq 12$, s $P$ SGA $\geq 3$, body surface area (BSA) $\geq 10 \%$

- Stratified by geographic region, body weight, and prior biologic us
- Coprimary endpoints were the proportion of patients who achieved

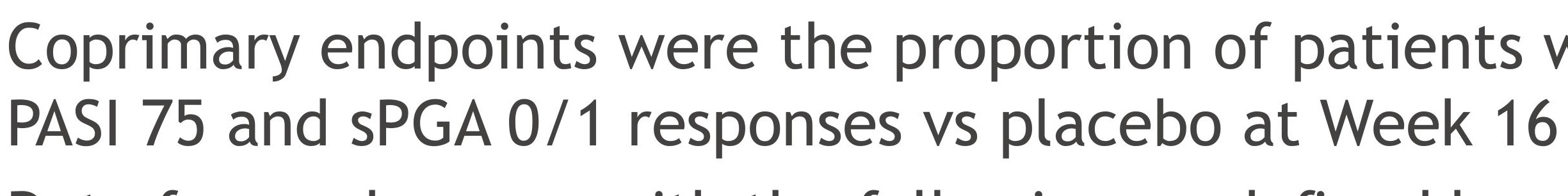

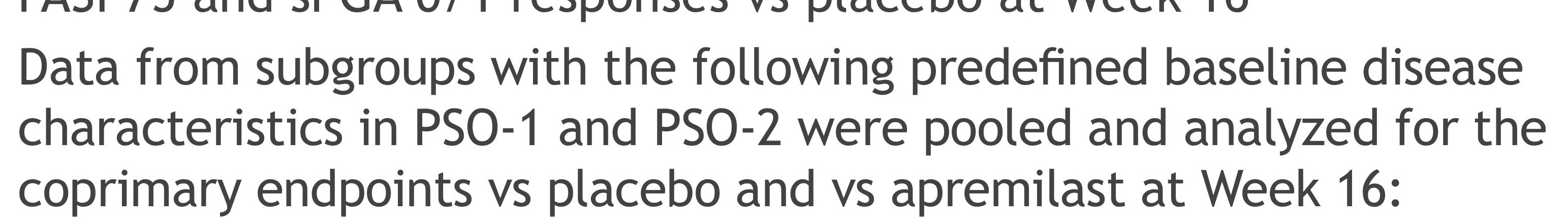

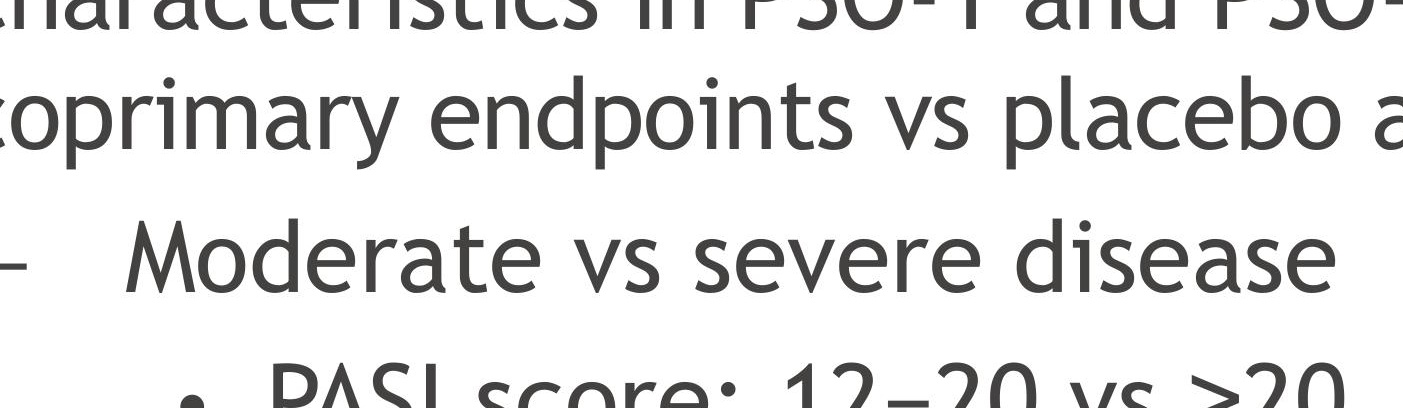

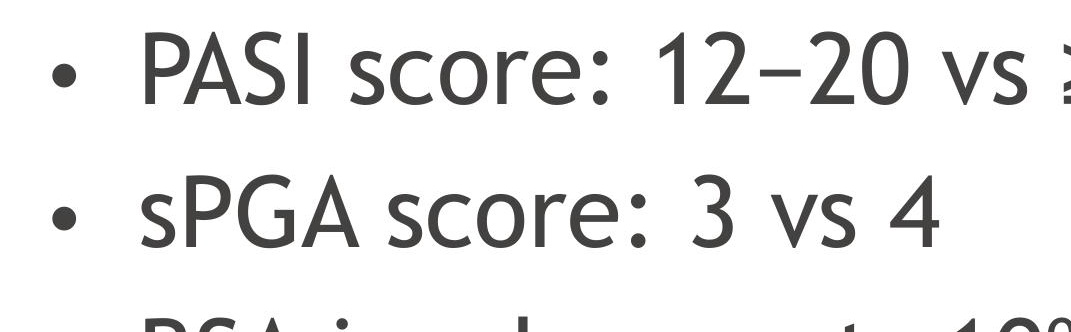

- BSA involvement: $10 \%-20 \%$ vs $>20 \%$
Disease duration: $<10$ y vs $>10$ y

Age at disease onset subgroups: $18 \mathrm{y}, 18-39 \mathrm{y}, 240 \mathrm{y}$

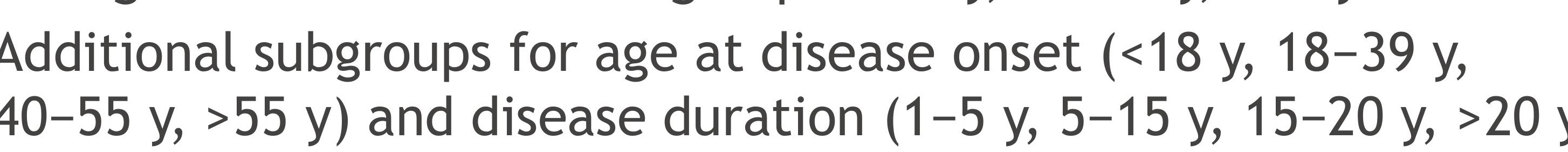

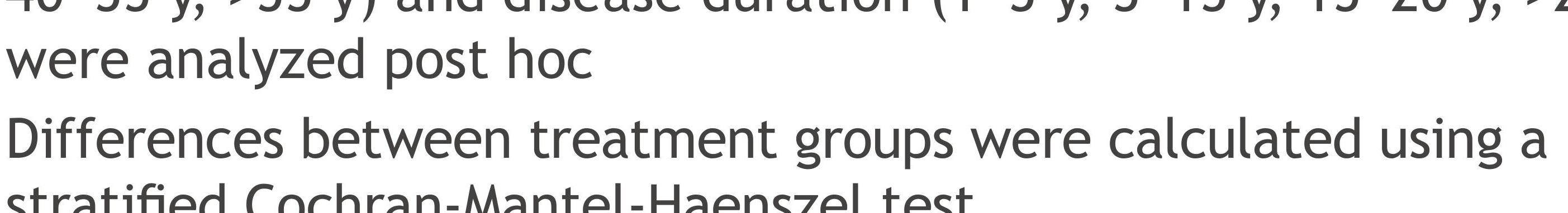
- Strtatified cochran-Mantel--Haenszel test
Missing data were imputed with nonresponder imputation

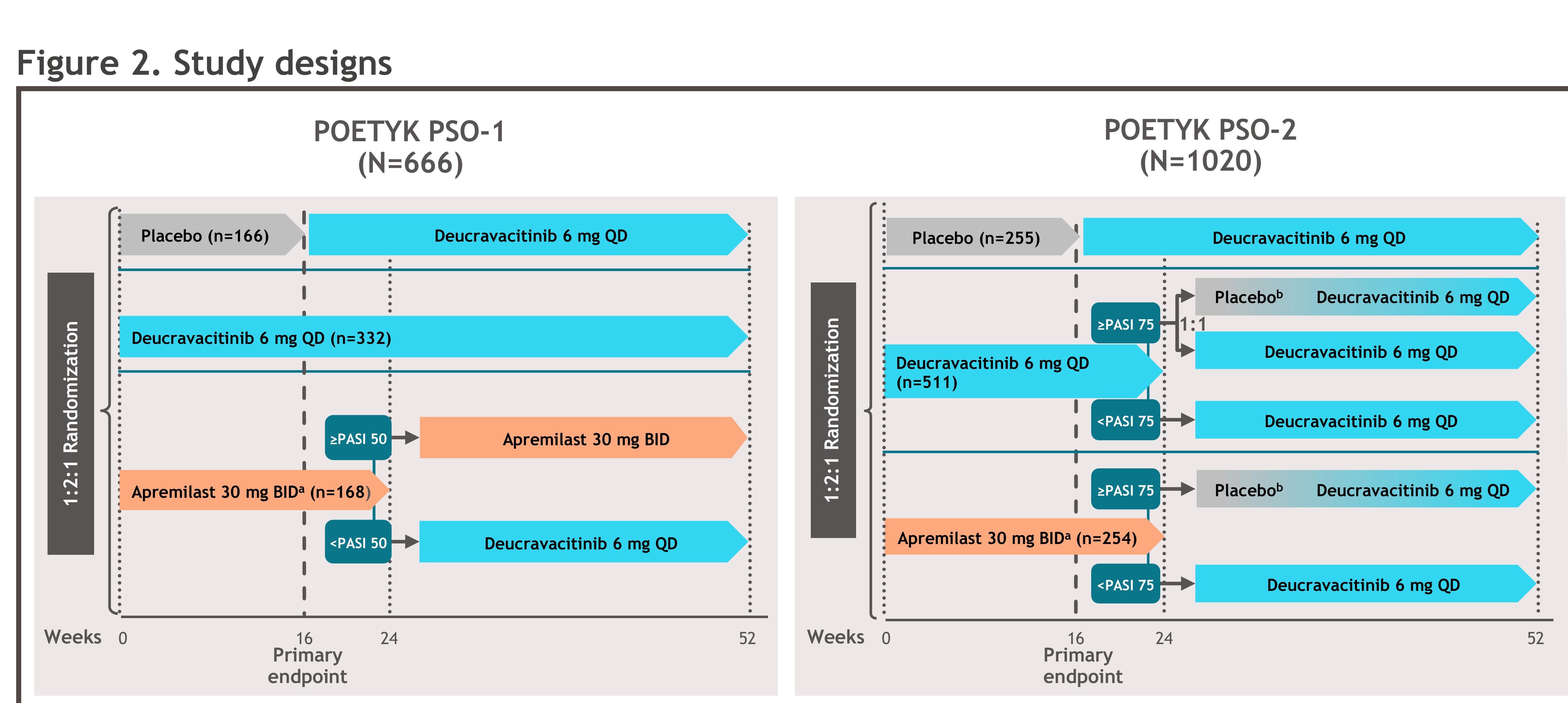

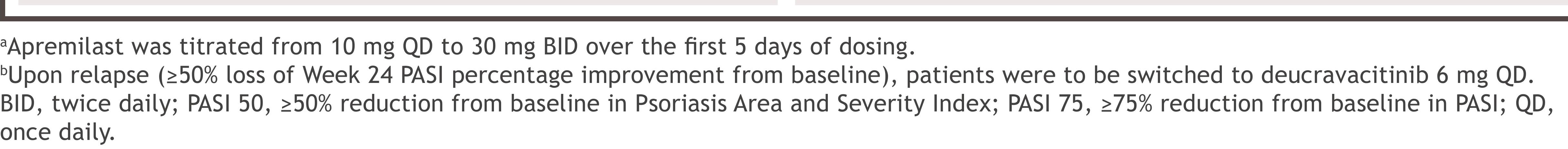

Results

Baseline patient demographics and disease characteristics

Baseline patient demographics and disease characteristics were largely similar across treatment groups in
pooled data from the 2 trinas (Table 1) Table 1. Baseline patient demograptis.

\begin{tabular}{|c|c|c|c|c|}
\hline & \multicolumn{4}{|c|}{ Pooled POETYK PSO-1 and PSO-2 } \\
\hline & 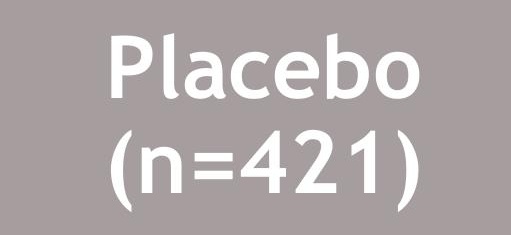 & 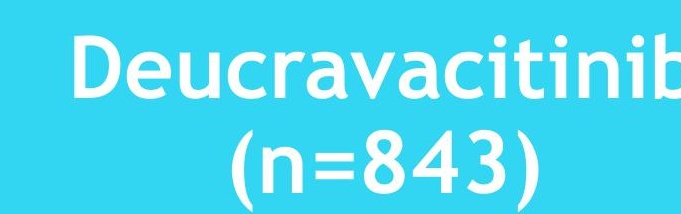 & 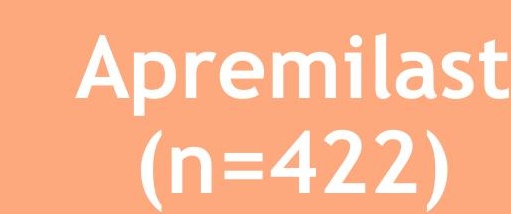 & $\underset{\substack{\text { Totat } \\
(n=1086)}}{n}$ \\
\hline Age, $y$, mean (SD) & $47.5(13.7)$ & 46.5 (13.5) & $45.7(12.8)$ & $46.6(13.4)$ \\
\hline Weight, kg, mean (SD) & $90.6(21.1)$ & 90.6(21.9) & $91.1(22.0)$ & 90.721 .77 \\
\hline Female, $n$ (⿻) & $127(30.2)$ & $277(32.9)$ & 155 (36.7) & $\begin{array}{l}5599(33.2) \\
\end{array}$ \\
\hline Race, $n$ (:) & & & & \\
\hline White & 360 (85.5) & 741 (87.9) & $368(87.2)$ & $146987.1)$ \\
\hline Asian & $42(10.0)$ & $83(9.8)$ & $40(9.5)$ & $1655(9.8)$ \\
\hline Other & $19(4.5)$ & $19(2.3)$ & $14(3.3)$ & 52 (3.1) \\
\hline 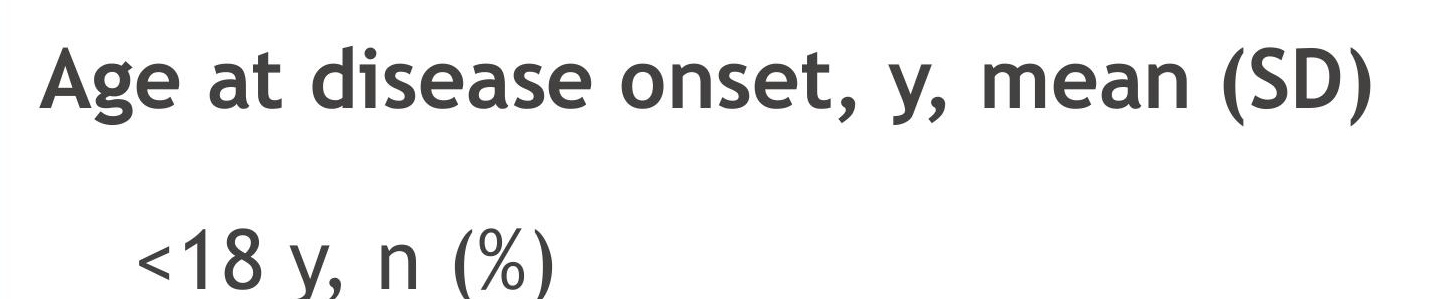 & $29.6(15.2)^{\prime \prime}$ & $28.8(14.9)$ & $28.1(14.7)$ & $\begin{array}{ll}28.8(14.9) \\
-4202050)\end{array}$ \\
\hline $18-39 y, n(\%)$ & $205(48.7)$ & 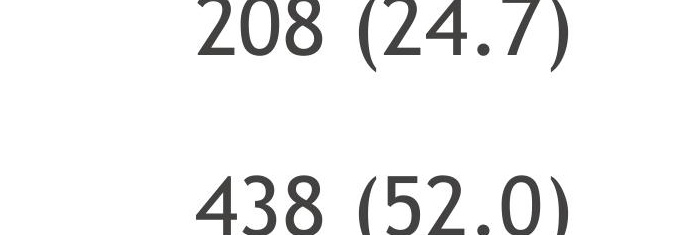 & $\begin{array}{l}\quad 112(26.5) \\
215(50.9)\end{array}$ & $858(50.9)$ \\
\hline$z 40 y, n(\%)$ & $113(26.8)$ & $197(23.4)$ & $95(22.5)$ & $405(24.0)$ \\
\hline Disease duration & 18.9 $(12.9)^{\mathrm{m}}$ & 18.6(12.7) & $18.5(12.1)$ & $18.6(12.6)$ \\
\hline$<10 y, n(8)$ & $199(28.3)$ & 261 (131.0) & $112(26.5)$ & $492(29.2)$ \\
\hline $210 y, n(x)$ & $301(71.5)$ & 582(69.0) & $310(77.5)$ & $1193(70.8)$ \\
\hline Prior system & & & & \\
\hline $\begin{array}{l}\text { Biologic } \\
\text { No prior sstemic therapy }\end{array}$ & $\begin{array}{l}146(34.7) \\
173(411)\end{array}$ & $295535.0)$ & $\begin{array}{l}145(34,4) \\
173(310)\end{array}$ & 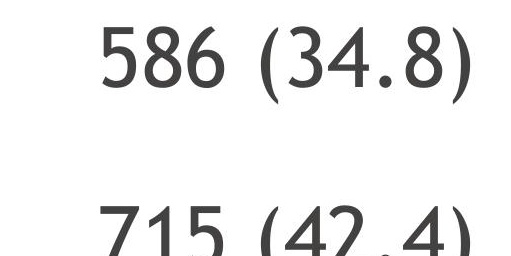 \\
\hline SPCA, n (\%) & & & & \\
\hline & $345(81.9)$ & $665(78.9)$ & $335(79.4)$ & $1345(79.8)$ \\
\hline $4=$ severe & $75(17.8)$ & $178(21.1 .1)$ & $87(20.6)$ & $340(20.2)$ \\
\hline PASI, mean (SD) (overall) & $20.9(8.6)$ & $21.1(8.0)$ & $21.6(8.6)$ & $21.2(8.3)$ \\
\hline 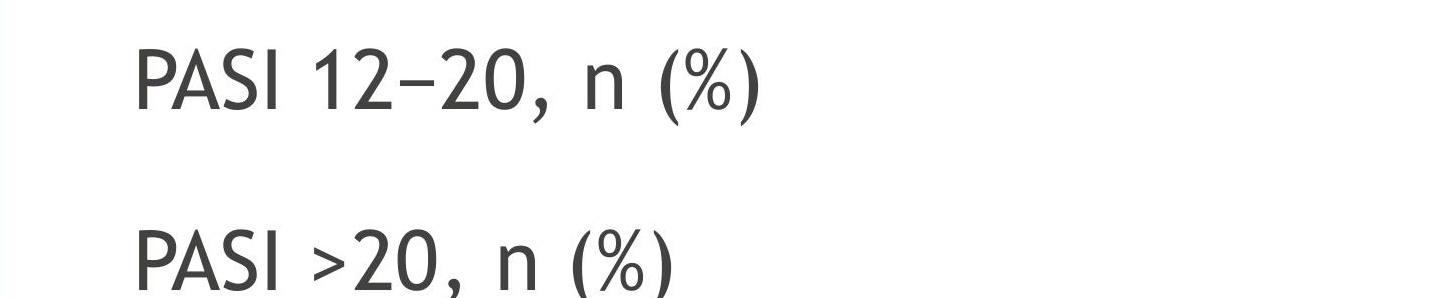 & $254(60.3)$ & 475 (56.3) & $241(57.1)$ & $970(57.5)$ \\
\hline BSA_mean (SD) (overall) & & & & \\
\hline BSA 10\%-20\%, n(s) & 226 (53.7) & 421 (49.9) & $200(47.4)$ & $847(50.2)$ \\
\hline $\mathrm{BSA}=20 \%, \mathrm{n},(8)$ & & & $222(52.6)$ & \\
\hline
\end{tabular}

Efficacy

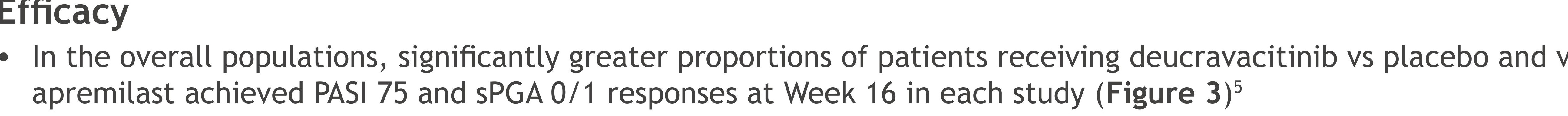
Figure 3. PASI 75 and SPGA 0/1 responses at Week 16 for PSO-1 and PSO-2

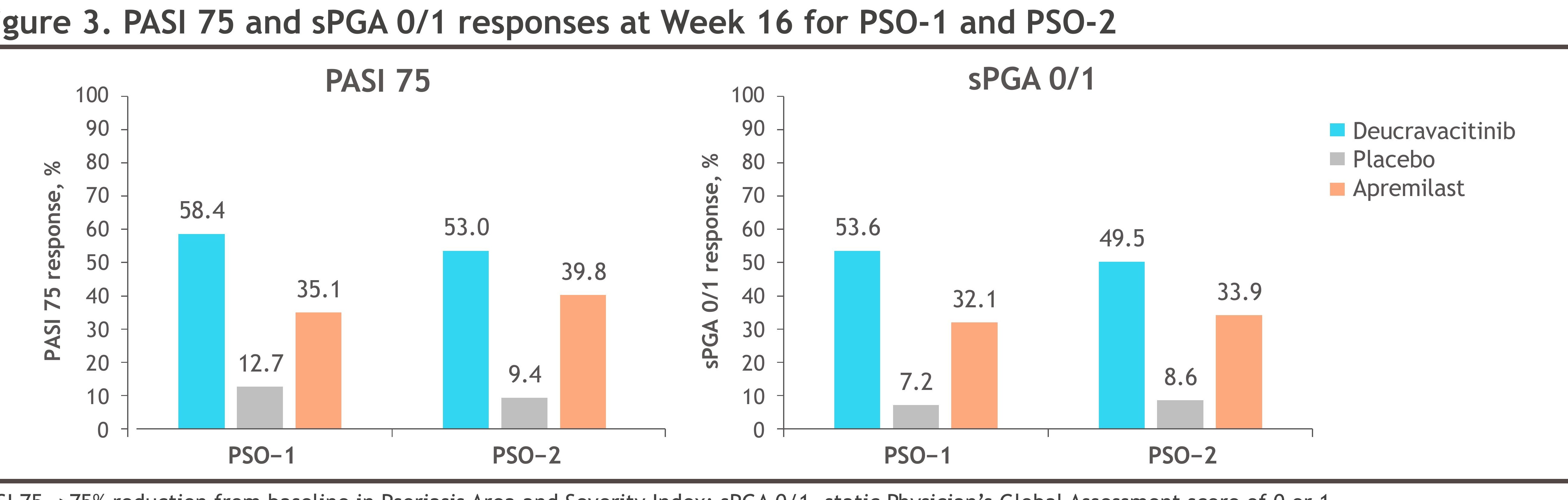

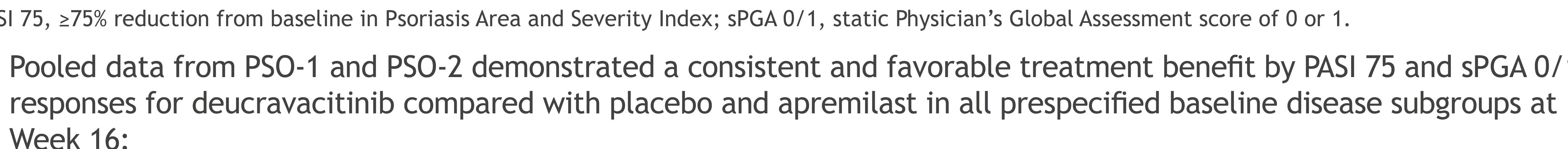
Disease severity by PASI, SPGA, and BSA

Disease duration
Age of disease onset fFigure 4 and Figure 50

Figure 4 . PASI 75 response at Week 16: PSO-1 and PSO-2 pooled analysis - efficacy of deucravacitinib

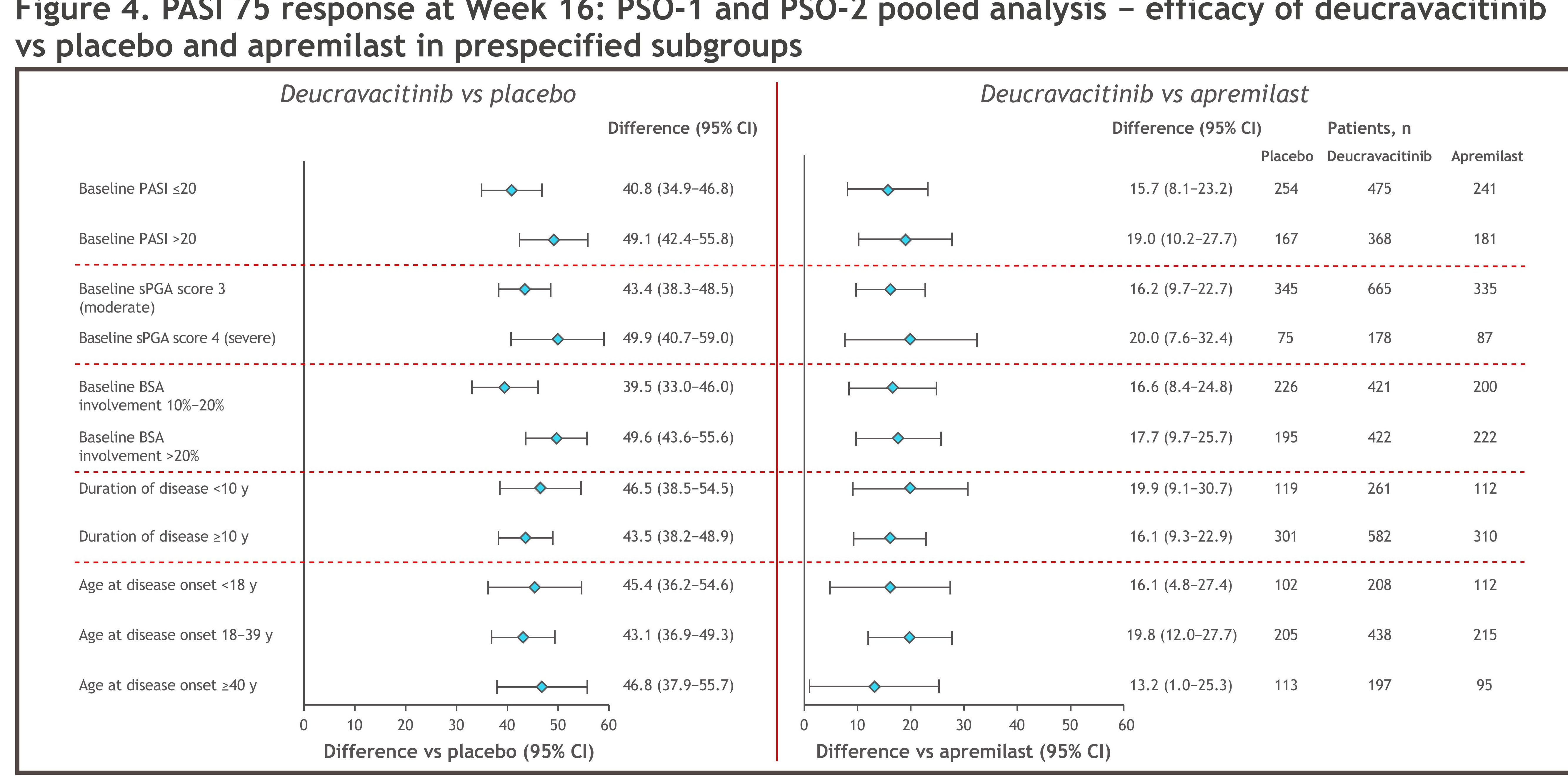

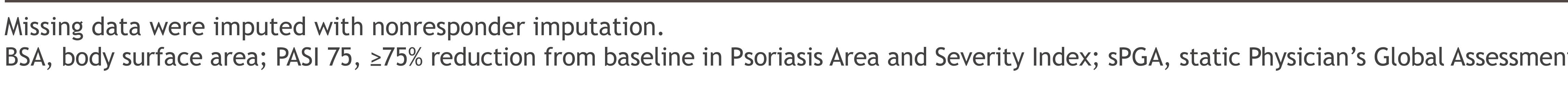

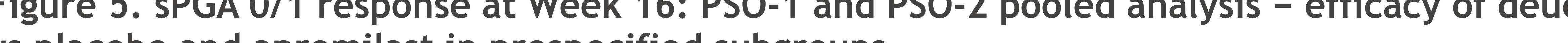

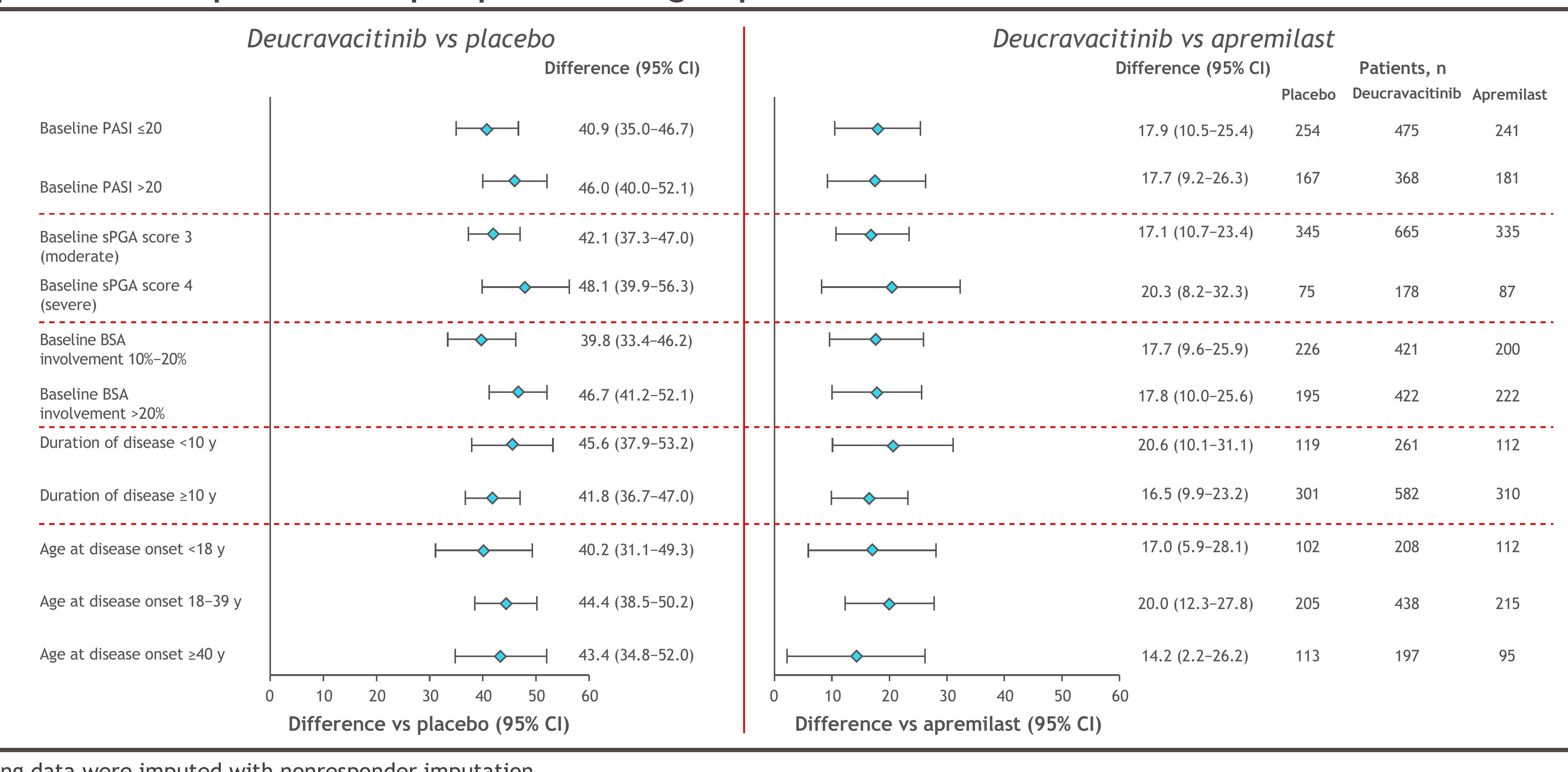

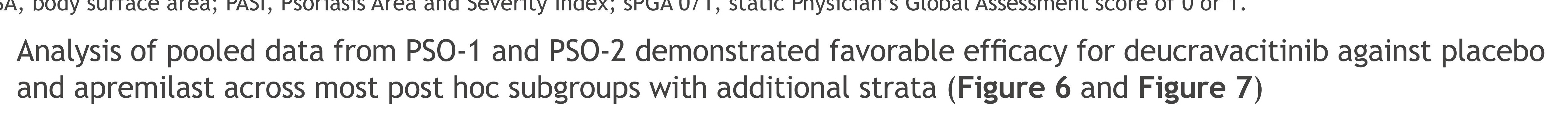

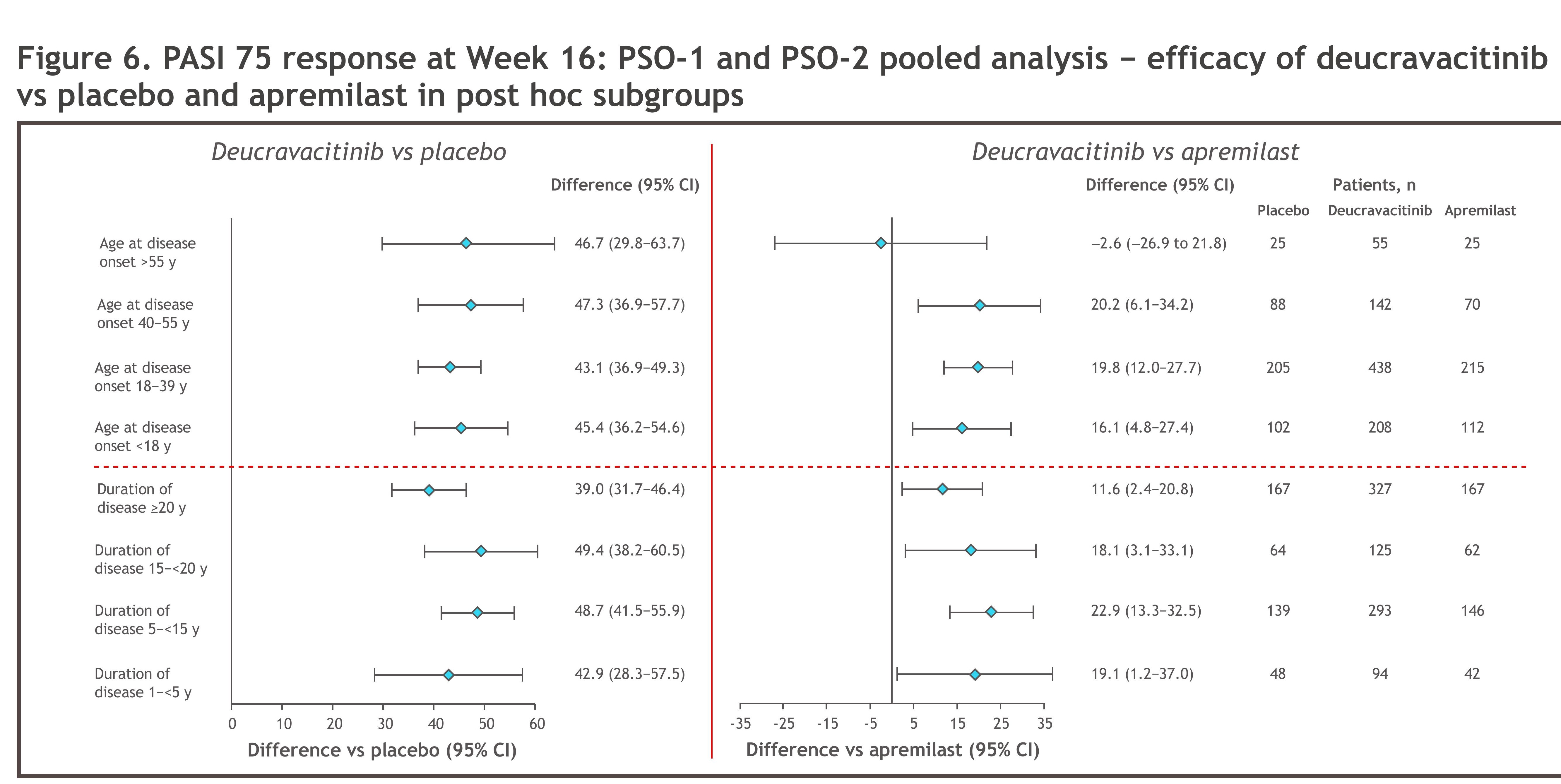

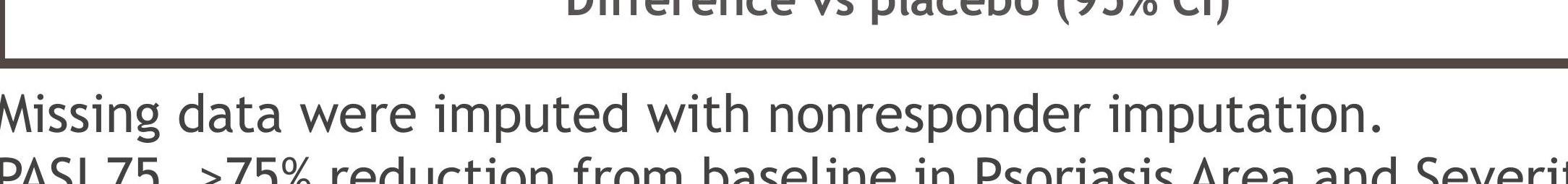

Figre 7 . SPGAO1 1 response at Wek 16: PSO-1 and PSO-2 pooled analysis - efficacy of deucravacitin Figure 7. SPGA $0 / 1$ response at Week 16 : PSO-1 a a d
vs placebo and apremilast in post hoc subgroups

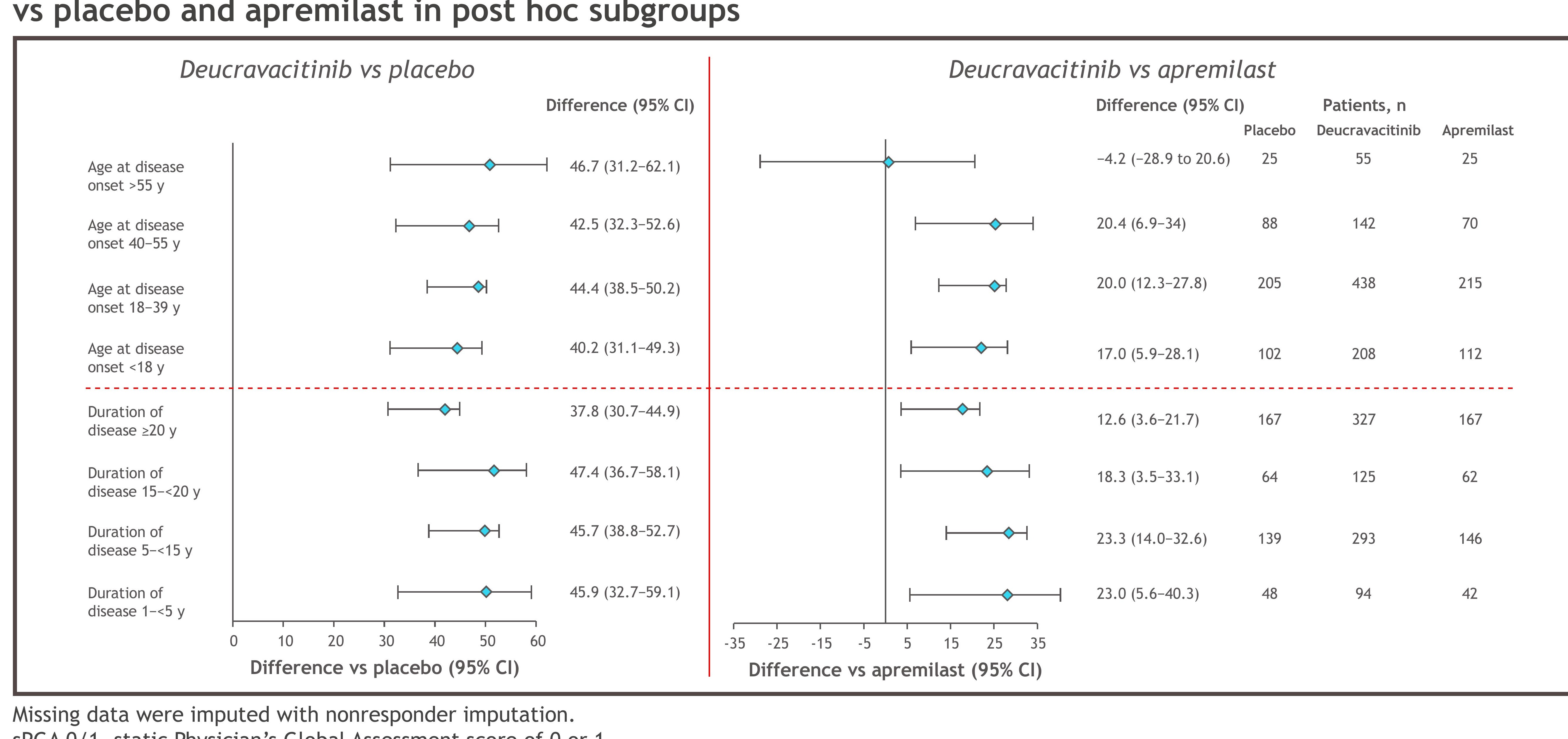

Conclusions

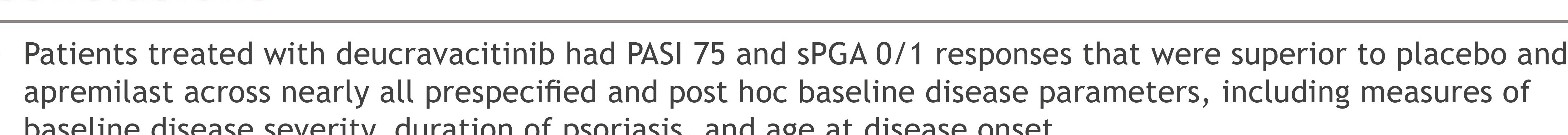

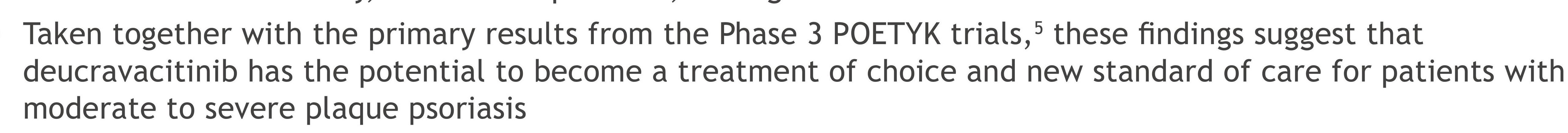
References

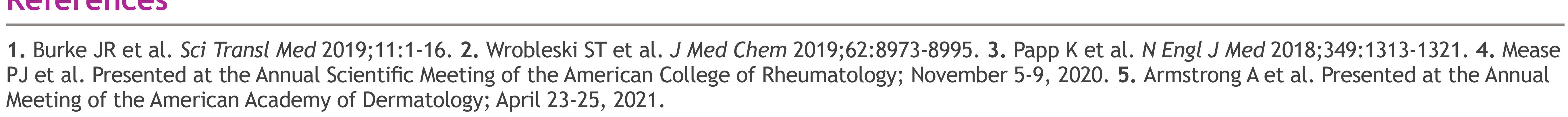

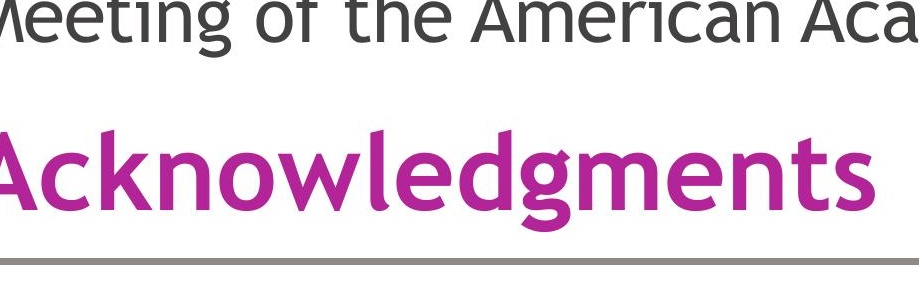

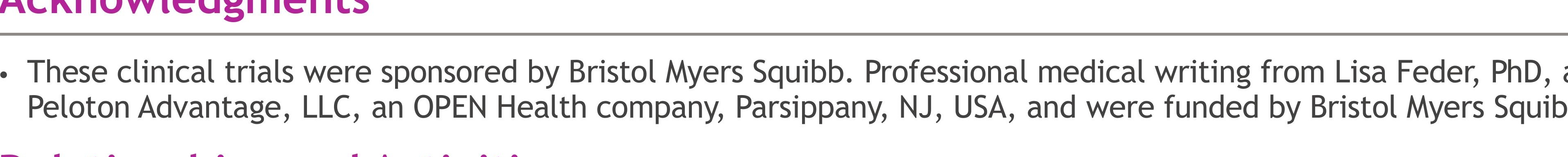

Relationships and Activities

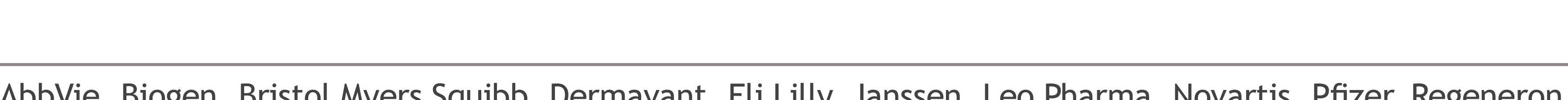

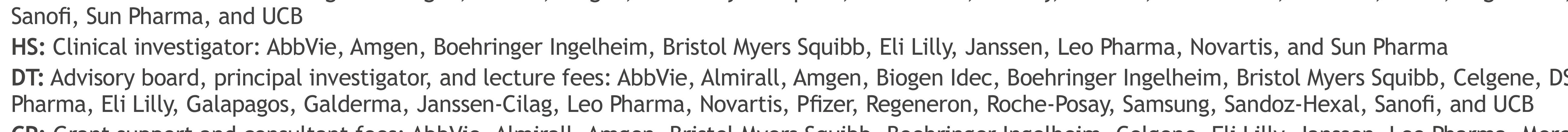

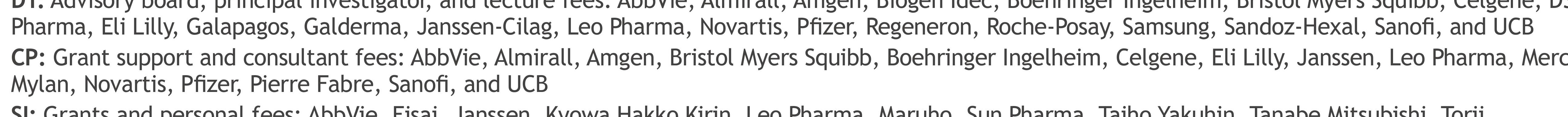

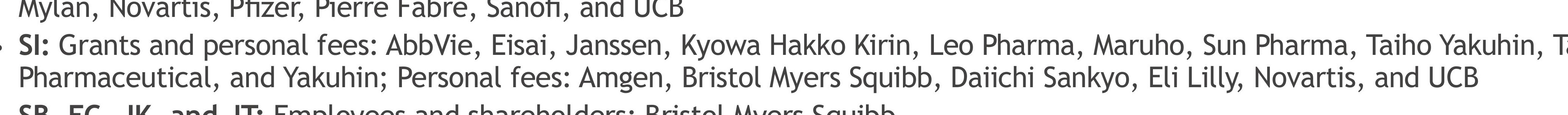

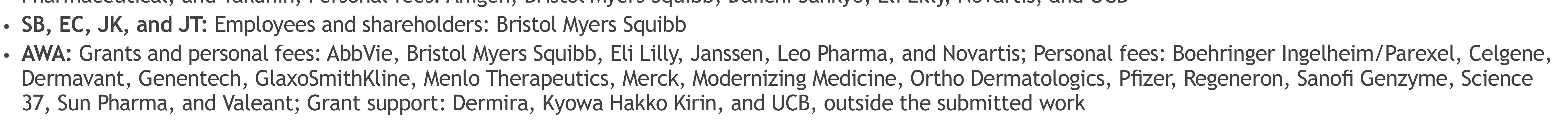

